\title{
Pyramidal and toroidal water drops after impact on a solid surface
}

\author{
By Y. RENARDY ${ }^{1}$, S. POPINET ${ }^{2}$, L. DUCHEMIN ${ }^{3}$, \\ M. RENARDY ${ }^{1}$, S. ZALESKI I C. JOSSERA N D ${ }^{3}$, \\ M. A. DRUMRIGHT-CLARKE ${ }^{1}$, D. R I CHAR D ${ }^{4}$, \\ C. CLANET AND D. QUÉRE ${ }^{6}$ \\ ${ }^{1}$ Department of Mathematics and ICAM, 460 McBryde Hall, Virginia Tech, Blacksburg, \\ VA 24061-0123, USA \\ ${ }^{2}$ National Institute for Water and Atmospheric Research, PO Box 14 901, Kilbirnie, \\ Wellington, New Zealand \\ ${ }^{3}$ Laboratoire de Modélisation en Mécanique, CNRS-UMR 7607, Université Pierre et Marie Curie, \\ 8 rue du Capitaine Scott, 75015 Paris Cedex 05, France \\ ${ }^{4}$ Department of Applied Mathematics and Theoretical Physics, University of Cambridge, Centre for \\ Mathematical Sciences, Wilberforce Road, Cambridge CB3 0WA, UK \\ ${ }^{5}$ Institut de Recherche sur les Phénomènes Hors Équilibre, UMR 6594 du CNRS, BP 146, \\ 13384 Marseille Cedex, France \\ ${ }^{6}$ Laboratoire de Physique de la Matire Condense, URA 792 du CNRS, Collège de France, \\ 75231 Paris Cedex 05, France
}

(Received 23 July 2002 and in revised form 26 December 2002)

Superhydrophobic surfaces generate very high contact angles as a result of their microstructure. The impact of a water drop on such a surface shows unusual features, such as total rebound at low impact speed. We report experimental and numerical investigations of the impact of approximately spherical water drops. The axisymmetric free surface problem, governed by the Navier-Stokes equations, is solved numerically with a front-tracking marker-chain method on a square grid. Experimental observations at moderate velocities and capillary wavelength much less than the initial drop radius show that the drop evolves to a staircase pyramid and eventually to a torus. Our numerical simulations reproduce this effect. The maximal radius obtained in numerical simulations precisely matches the experimental value. However, the large velocity limit has not been reached experimentally or numerically. We discuss several complications that arise at large velocity: swirling motions observed in the cross-section of the toroidal drop and the appearance of a thin film in the centre of the toroidal drop. The numerical results predict the dry-out of this film for sufficiently high Reynolds and Weber numbers. When the drop rebounds, it has a top-heavy shape. In this final stage, the kinetic energy is a small fraction of its initial value.

\section{Introduction}

The hydrophobicity of solids can be dramatically enhanced by texturing the surface, as in the case of super-hydrophobic surfaces on which water drops behave like pearls. These surfaces can be natural, an example being the leaves of gingko biloba, which is used in urban areas for its capacity to resist pollution (Neinhuis \& Barthlott 1997). They can also be manufactured for various purposes: water or frost-repellent surfaces, 
waterproof textiles, self-cleaning substrates, and so on (see Nakajima, Hashimoto \& Watanabe 2001 for a recent review). In each case, the solid surface is decorated with a rough microstructure, typically at length scales of $100 \mathrm{~nm}$ to $10 \mu \mathrm{m}$, and then coated with wax so that the liquid sits on top of the rough texture without penetrating into it. Thus, the liquid lies on a patchwork of solid and air, which enables very high contact angles (such as 160-170 ) (Bico, Marzolin \& Quéré 1999). In the experiments reported in this paper, the solid surface is made super-hydrophobic by deposition and adhesion of a porous powder (lycopodium grains) treated with silanes (to reinforce the hydrophobicity). The results do not depend on the solid: other super-hydrophobic solids such as natural leaves were also tested, giving the same results. The drops impacting on the surface are generated from a pipette, so that their size is selected by the diameter of this pipette. The speed of impact is controlled by the height from which the droplets are ejected.

The impact of water drops on these surfaces exhibits quite unusual features, a noteworthy one being the possible rebound of the entire drop (Hartley \& Brunskill 1958; Richard 2000; Richard \& Quéré 2000). This elastic behaviour is favoured by the film of air below the drop, which eliminates direct liquid-solid contact; this avoids the dissipation which usually takes place along moving contact lines. After the drop hits the surface, it spreads because of its kinetic energy. The diameter passes through a maximum, and then the drop recoils. The entire drop lifts off, in contrast to the case of a normal (non-superhydrophobic) surface, for which a portion of the splatted drop remains on the surface (cf. figure 4 of Fukai et al. 1993; Pasandideh-Fard et al. 1996; Bussmann, Mostaghimi \& Chandra 1999).

The behaviour during impact depends mainly on the impact velocity $V$, or more precisely on the Weber number We, which is the ratio of kinetic energy to surface energy:

$$
W e=\frac{\rho V^{2} R}{\gamma} .
$$

Here, $R$ denotes the radius of a sphere with the same volume as the drop, and $\rho$ and $\gamma$ denote the density and surface tension of the liquid, respectively.

For $W e<1$, drop deformation during impact is small, and the drop retains an oval shape (Richard \& Quéré 2000). For $W e>1$, the deformation becomes significant (of the order of the drop radius), and the drop spreads over the solid, with a well-defined maximal extension. Above a threshold Weber number (We $\sim 4)$, capillary waves travel to the top without being damped, resulting in a pyramid structure with several steps (figure 1). This structure can also occur for a normal solid surface (cf. experimental photos of Martin 1991 and Pasandideh-Fard et al. 1996); indeed, it should exist on normal solids because it takes place in the inertial regime of spreading, where the wetting properties do not play a major role. Moreover, we observe similar structures for drops of low-viscosity silicone oil, impacting at $W e \sim 5$, on glass substrates that they completely wet. The case of a liquid substrate is quite different, as shown by Thoroddsen \& Takehara (2000), leading to a bell shape with concentric surface disturbances, which eject droplets from the centre before coalescing with the bulk fluid.

A primary goal of this paper is to describe the appearance and evolution of the pyramid structure. Scaling arguments allow us to predict the interval in velocity in which it should be observed. The capillary wave of maximal amplification has a phase 

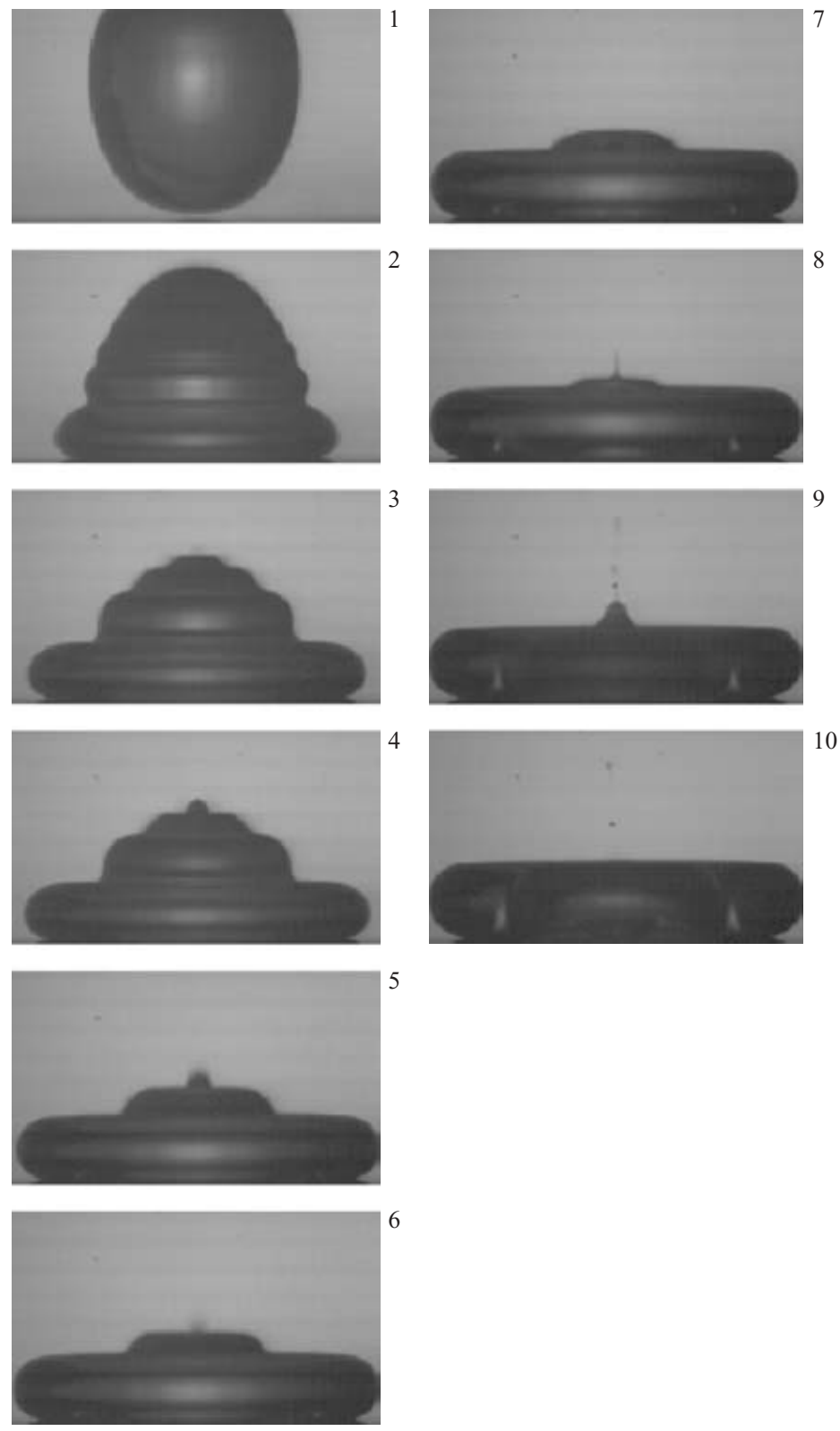

FiguRE 1. Structures formed during the impact of a drop of radius $R=1.75 \mathrm{~mm}$ at velocity $V=41 \mathrm{~cm} \mathrm{~s}^{-1}$. The timings from frame 1 are $2.7,4.5,4.9,5.9,6.1,6.3,6.7,7.1,8.0 \mathrm{~ms}$.

speed equal to the impact speed; hence, its wavelength $\lambda$ is

$$
\lambda=\frac{\gamma}{\rho V^{2}}
$$

For the parameters in figure $1\left(V=40 \mathrm{~cm} \mathrm{~s}^{-1}, \gamma=72.8 \mathrm{dyn} \mathrm{cm}^{-1}\right.$ at $20^{\circ} \mathrm{C}, \rho=$ $1 \mathrm{~g} \mathrm{~cm}^{-3}$ ), we obtain $\lambda=0.5 \mathrm{~mm}$, which is roughly the height of the layers. More 
generally, waves can only be observed if $\lambda<R$, which immediately yields

$$
W e>1 \text {, }
$$

Figure 1 shows that steps in the staircase subsequently empty into each other, and the oscillation of the top can lead to the formation of a small cavity of air. The collapse of this cavity eventually produces the eruption of a very thin jet (8th snapshot in figure 1). This is reminiscent of simulations of a gas bubble bursting at a free surface (Boulton-Stone \& Blake 1993; Oguz 1998; Duchemin et al. 2001). Later, the whole drop retracts because of the hydrophobicity of the substrate, and finally lifts off.

When the impact speed is slightly higher, dry-out can occur at the centre: the outward spreading of the drop progresses to the point where a dry spot forms at the centre of the original impact and the drop takes a toroidal shape. For a normal solid, a similar situation is observed (cf. figure 3 of Fukai et al. 1993), although a thin layer of liquid remains at the centre. Whether dry-out is achieved or not can be an important practical piece of information, and we discuss it in $\S 3$.

For still higher impact speeds, the pyramid is no longer observed, and is replaced by a fried egg structure. The distance $\ell$ of decay of a wave of wavelength $\lambda$ propagating at speed $V$ can be written on dimensional grounds:

$$
\ell \sim \frac{\rho \lambda^{2} V}{\eta},
$$

where $\eta$ is the viscosity. In order to observe the pyramid structure, $\ell$ must be much larger than the radius of the drop. The condition $\ell>R$ with (1.4), and substitution of (1.2) for $\lambda$ yields $\rho R \eta V^{3} / \gamma^{2}<1$. In terms of $W e$ and the capillary number

$$
C a=\frac{\eta V}{\gamma},
$$

this yields the criterion

$$
\text { We } C a<1 .
$$

For the drop of radius $R=1.75 \mathrm{~mm}$, the criterion is verified since $W e C a \approx 0.02$. This criterion together with the requirement of large We yields the window of velocities for the likely occurrence of a staircase structure:

$$
\left(\frac{\gamma}{\rho R}\right)^{1 / 2}<V<\left(\frac{\gamma^{2}}{\rho \eta R}\right)^{1 / 3} .
$$

For a drop of water of $R=1 \mathrm{~mm}$, this gives speeds between 26 and $170 \mathrm{~cm} \mathrm{~s}^{-1}$. At the higher end of this range, the pyramid gives way to the fried egg structure.

The numerical algorithm is summarized in $\S 2$. An axisymmetric finite volume formulation is used on a fixed grid, and a front-tracking method is developed for the free surface, which is approximated by cubic splines that connect marker points. This method allows us to deal with large drop deformations. The sections thereafter are devoted to the results. First, $\S 3$ describes conditions for generating a toroidal drop with a dry spot at the centre. Secondly, $\S 4$ addresses drop evolution during impact, with special focus on the regime where liquid pyramids can be obtained. Thirdly, we discuss the evolution of kinetic energy in $\S 5$. Finally, $\S 6$ is a discussion of the maximal spreading diameter upon impact. 


\section{Numerical method}

The computational domain is a square in the $(r, z)$-plane. We use a fixed Cartesian mesh of square cells. The velocity components and pressure are evaluated at centres of edges or cells with the MAC grid. The front-tracking method is the free-surface marker method described in detail in Popinet \& Zaleski (2002) and we sketch it here.

\subsection{Governing equations}

A finite volume formulation is used for the axisymmetric Navier-Stokes equations, which are converted to an integral form over each cell. That is, the momentum equation

$$
\rho\left(\frac{\partial \boldsymbol{u}}{\partial t}+(\boldsymbol{u} \cdot \nabla) \boldsymbol{u}\right)=-\nabla p+\operatorname{div} \boldsymbol{S}
$$

is converted to the integral form

$$
\int_{\Omega} \rho \boldsymbol{u}_{t} \mathrm{~d} V+\int_{\partial \Omega} \rho \boldsymbol{u}(\boldsymbol{u} \cdot \boldsymbol{n}) \mathrm{d} S=\int_{\partial \Omega}(\boldsymbol{S} \cdot \boldsymbol{n}-p \boldsymbol{n}) \mathrm{d} S .
$$

Here $\boldsymbol{S}$ denotes the viscous stress tensor. This equation is then discretized within each cell. When the cell is cut by the interface, the surface integrals involve a portion over the interface. At the free surface, shear stress is required to be zero, and the jump in the normal stress is balanced by the surface tension force. That is, we have

$$
\boldsymbol{s} \cdot \boldsymbol{n}-p \boldsymbol{n}=-\sigma \kappa \boldsymbol{n},
$$

where $\sigma$ is the surface tension coefficient and $\kappa$ is the curvature.

A projection method is used to separate the update of the velocity from the computation of the pressure. In the projection, we first calculate an intermediate velocity field $\boldsymbol{u}^{*}$ from a discretized version of the equation

$$
\int_{\Omega} \rho \frac{\boldsymbol{u}^{*}-\boldsymbol{u}^{n}}{\Delta t} \mathrm{~d} V+\int_{\partial \Omega} \rho \boldsymbol{u}^{n}\left(\boldsymbol{u}^{n} \cdot \boldsymbol{n}\right) \mathrm{d} S=\int_{\partial \Omega_{1}} \boldsymbol{S} \cdot \boldsymbol{n} \mathrm{d} S-\int_{\partial \Omega_{2}} \sigma \kappa \boldsymbol{n} \mathrm{d} S .
$$

Here $\partial \Omega_{1}$ is the part of $\partial \Omega$ which is in the interior of the flow domain, and $\partial \Omega_{2}$ is the part on the interface. At the second step of the projection, we set

$$
\int_{\Omega} \rho \frac{\boldsymbol{u}^{n+1}-\boldsymbol{u}^{*}}{\Delta t} \mathrm{~d} V=-\int_{\partial \Omega_{1}} p \boldsymbol{n} \mathrm{d} S
$$

and $p$ is determined from solving a Poisson-type equation which exploits the fact that $\boldsymbol{u}^{n+1}$ ought to be divergence-free. A multigrid scheme is used to solve this.

\subsection{Boundary and initial conditions}

The boundary condition at the bottom wall of the computational box is a free-slip condition. This was chosen since it is generally expected that friction is weak or vanishing during impact, with possibly thin air films forming between the surface and the liquid. For the bottom wall condition, a continuous marker chain is used for the free surface: the marker chain is closed and wraps around the bottom of the drop, where, after impact, it lies 'flat' along the bottom surface. Physically, this is an interface lying exactly parallel to the bottom wall. This reduces to imposing a $180^{\circ}$ contact angle at the point where the interface 'detaches' from the solid boundary, i.e. a perfectly non-wetting surface. The features observed in laboratory experiments are not sensitive to small changes in the contact angles, and the dynamic contact angle is always found to saturate at $180^{\circ}$ (see figure 1 for example). 
To be able to calculate finite difference derivatives of the velocity near the interface, we need to extrapolate the velocity outside the flow region. This is done as follows. First, the cubic spline approximation to the surface position is used to calculate the normal pointing outwards from the liquid. Secondly, the velocities at the nodes within the liquid in this neighbourhood are extrapolated linearly to nodes in the air by a least-squares fit subject to vanishing shear stress.

In figure 1, the drop is generated by dripping. As the drop falls, it may oscillate. The main features of the pyramid and capillary waves are not sensitive to the initial conditions. Although not impossible, calculations of the moment of impact are tedious to set up and computationally expensive. Thus our calculations are done from an initial condition where the droplet is already touching the surface.

\subsection{Tracking the free surface}

The reader is referred to Popinet \& Zaleski (2002) for details. The surface tension term is computed using cubic spline curves. The markers are advected by the interpolated velocity field calculated on the fixed grid.

The front-tracking component is summarized as follows. The interface is represented using an ordered list of marker particles $\left(x_{i}, y_{i}\right), 1 \leqslant i \leqslant N$. A list of connected polynomials $\left(p_{i}^{x}(s), p_{i}^{y}(s)\right)$ is constructed using the marker particles and gives a parametric representation of the interface, with $s$ an approximation of the arclength. Both lists are ordered and thus identify the topology of the interface.

\subsubsection{Advecting the points}

The first step in the algorithm is the advection of the marker particles. A bilinear interpolation of the velocity field is used. The marker particles are advected in a Lagrangian manner with a first-order explicit scheme. Once the points have been advected, we then reconstruct the parametric representation of the interface using cubic splines.

\subsubsection{Redistribution}

As the interface evolves, the markers drift along the interface following tangential velocities and we may need more markers if the interface is stretched by the flow. We then need to redistribute the markers in order to ensure a homogeneous distribution of points along the interface. This is done at each time step. This is similar to the approach of boundary integral codes that also use arclength parameterization, cubic splines and redistribution of nodes.

\section{Critical curve for dry-out}

Figure 2 shows experimental photographs for a typical dry-out sequence (note the hole in the middle of the torus, which is clearly visible on the third picture of the sequence). For experimentalists, it is convenient to be able to predict whether a dry spot would emerge upon impact.

Our simulations are conducted in a computational box $14 / 3 \simeq 4.67 \mathrm{~mm}$ square with a $256 \times 256$ grid. Initially, the droplet is perfectly spherical and touches the ground. We define a Reynolds number:

$$
R e=\frac{V R \rho}{\eta}, \quad W e=C a R e .
$$



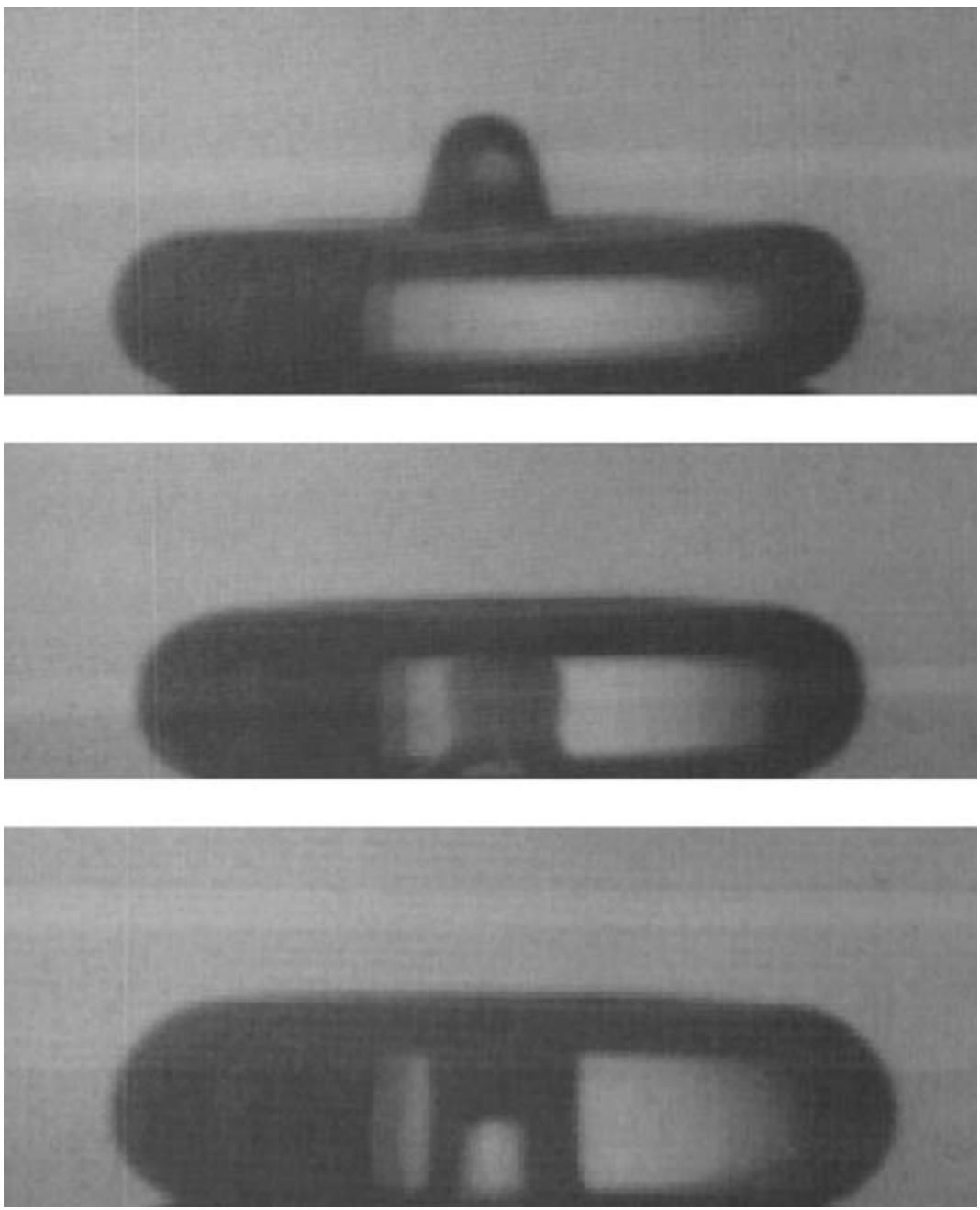

FIGURE 2. Photograph of a typical dry-out sequence. Experiment performed with a water drop for which $D=4 \mathrm{~mm}$, and $U=14 \mathrm{~cm} \mathrm{~s}^{-1}$ so that $W e=1.1$.

The effect of gravity is included in our calculations, but it is not an important factor during the impact phase. Figure 3 shows the critical values of Weber and Reynolds numbers for which the drop becomes a torus after impact. The intersection of the bottom wall with the axis of symmetry is 'Wet' for a spherical topology and 'Dry' for a toroidal one. The formula shown in the figure for the curve is an empirical fit.

At high Reynolds numbers, viscous effects are small and the topology is determined by a competition between just inertia and surface tension. Thus, the Weber number becomes the only dimensionless parameter and the graph asymptotes to $W e=$ constant, independent of the Reynolds number. On the other hand, at low Reynolds numbers, viscous effects are important and, therefore, a combination of both We and Re determines the critical curve.

For a given experimental set-up, figure 3 guides the selection of the drop velocity to achieve the desired condition. For example, in the development of technology for liquid-metal microdroplet deposition (Fukai et al. 1995), the 'wet' condition is desired. 


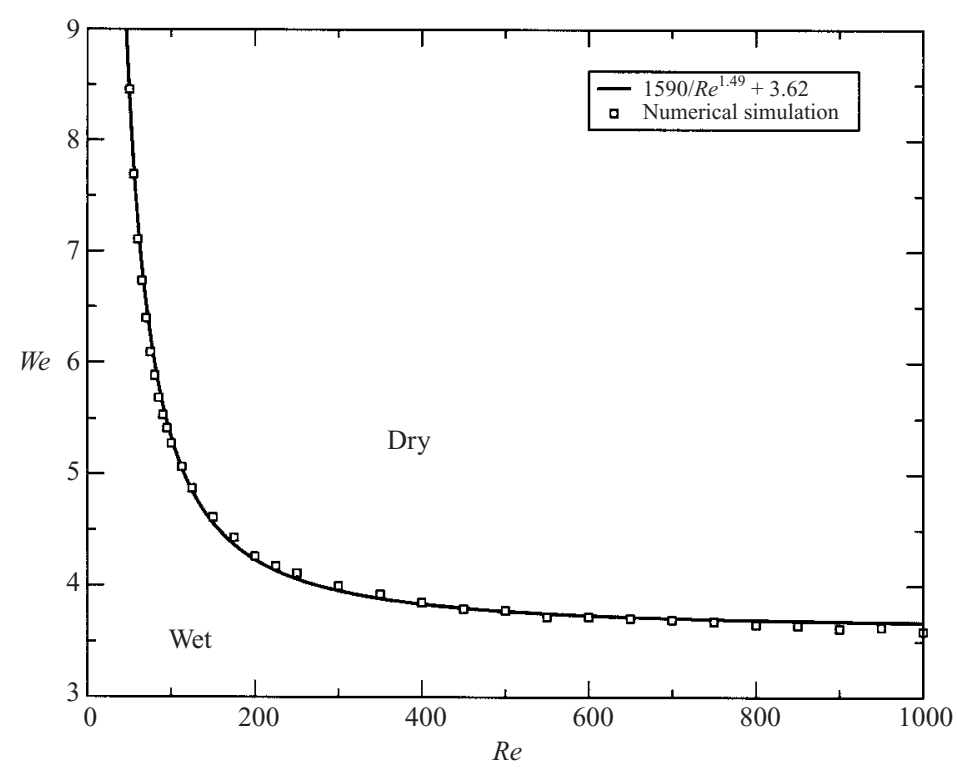

FIGURE 3. Curve delineating conditions for dry-out. Initial drop is spherical.

\section{Drop evolution}

For the results reported here, the computational box and grid are as in the previous section. The density of the liquid is $1 \mathrm{gm} \mathrm{cm}^{-3}$, and the viscosity is $1 \mathrm{cP}$. The surface tension is $72.8 \mathrm{dyn}^{-1}$.

We begin by showing in figure 4 the corresponding simulation for the experimental results of figure 1 displayed in $\S 1: 2.7 \mathrm{~ms}$ (between photo 1 and 2), 1.8 (between 2 and 3), etc. The initial shape is approximated well by a linear mode of oscillation of a drop released from a nozzle, namely mode 2 . The distance from the centre of the drop to the free surface is then

$$
r(\theta)=R_{0}\left(1.0+\epsilon Y_{2}(\theta)\right),
$$

where $Y_{2}(\theta)$ is a spherical harmonic of order 2 (Lamb 1932; Plesset \& Prosperetti 1977). This is used with $\epsilon=0.29$ deviation. Although we acknowledge that the initial shape of the drop may be due to the mode 2 oscillation, we do not attempt to include the velocity of the oscillations in our computations, because we do not know at what phase of the oscillation the drop is at impact. If the drop is at maximum distortion, the velocity is zero in the linear approximation. We can estimate the order of magnitude of the velocity of the oscillations. According to Lamb (1932), the frequency for a water drop is

$$
\omega=24.3 a^{-3 / 2},
$$

where $a$ is the radius of the drop (all numbers are in CGS units). For a maximum radial distortion of $\epsilon a$, we therefore find a maximum speed of

$$
u_{m}=24.3 \epsilon a^{-1 / 2} \text {. }
$$

With $\epsilon=0.29$ and $a=0.175$, this yields $u_{m}=16.85$. This speed, however, would be attained at the time when the distortion is zero and the drop is spherical. We believe that the drop at impact is actually close to maximum distortion and, therefore, the speed due to the oscillation at the time of impact is small relative to the impact speed. 
1
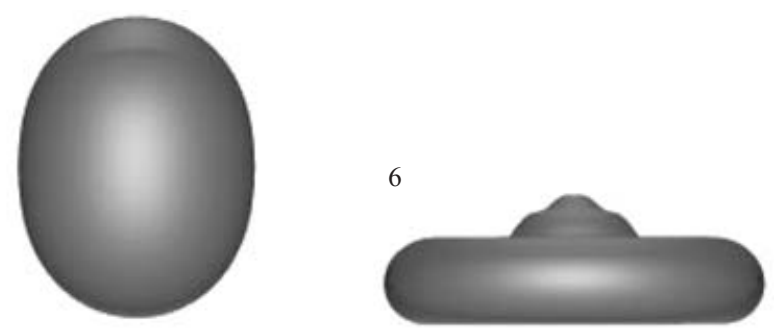

2

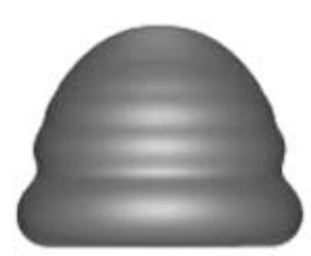

7

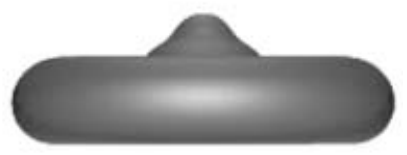

3

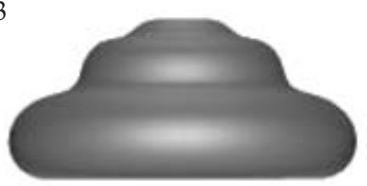

8

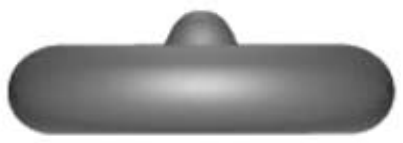

4

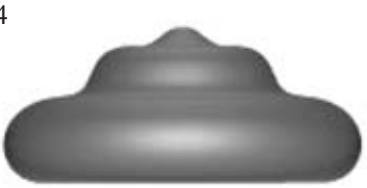

9

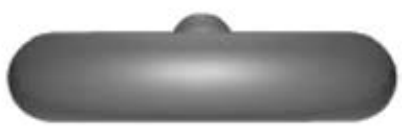

5

10
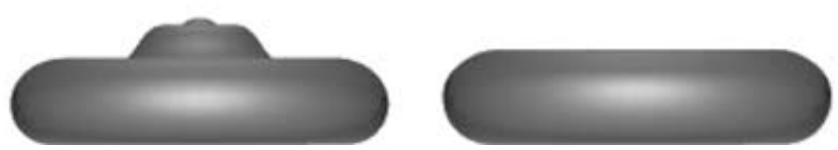

FiguRE 4. Direct numerical simulations. Initial drop is mode 2, with $R_{0}=1.75 \mathrm{~mm}$ and $\epsilon=0.29$ in equation (4.1). Initial velocity $V=0.41 \mathrm{~m} \mathrm{~s}^{-1}$. Timings between frames are the same as those of the experiment in figure 1.

Our numerical results, shown in figure 4, capture the main features of figure 1. We have conducted a spatial convergence test for these results and show in figure 5 the interface positions through a cross-section for the $256 \times 256$ case and the refined $512 \times 512$ case. These show that the former captures the qualitative features, while the latter accentuates the fine features such as the small capillary waves at the top. 
(a)
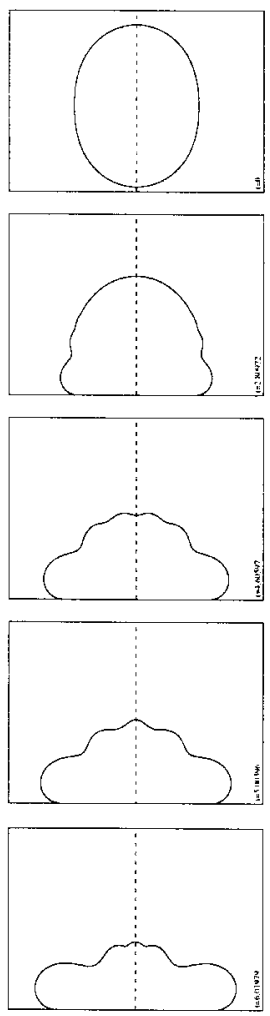
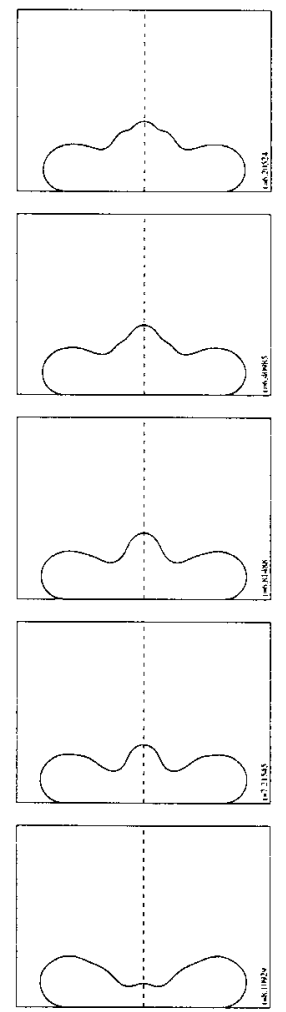
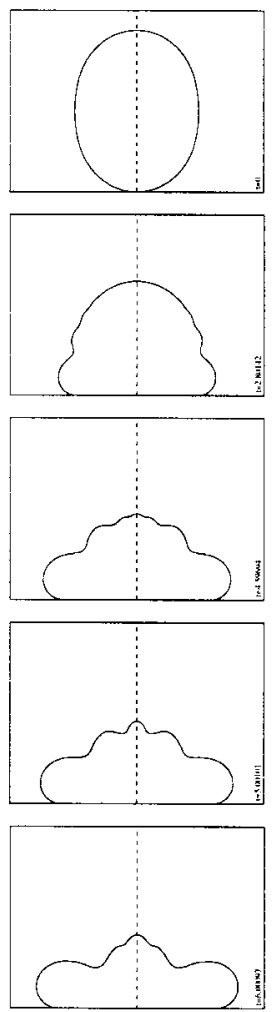

(b)
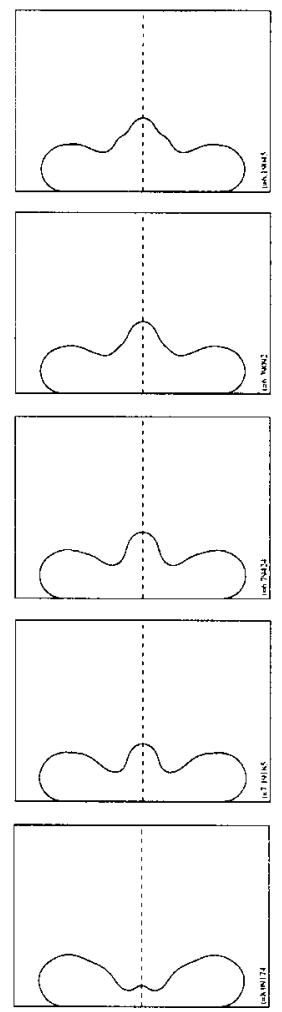

FIGURE 5. Spatial convergence test corresponding to figure 4. (a) $256 \times 256$ and (b) $512 \times 512$.

Figure 6 shows the contours for the pressure field corresponding to four of the stages in figure 4 . We see that the pyramidal steps are not flat but rimmed, as verified experimentally by taking photographs at an angle to the drop. These contour lines are at regular spacings of the pressure values. Clearly, the maximum values evolve with time toward the top of the pyramid.

\section{Kinetic energy evolution}

During the formation of the pyramid, the flow is upwards at the bottom centre of the drop, and downwards at the top centre. By the time the drop deforms to a torus, the motion is mainly a swirling motion in the $(r, z)$-plane. The evolution of kinetic energy is shown in figure 7 . When the drop collapses, kinetic energy is converted to surface energy which is partly in the form of capillary waves. The capillary waves travel up the drop, forming the pyramid. They meet at the top of the pyramid, forming a nipple. Thereafter, the centre of the drop continues its descent. At this point, the kinetic energy is at its minimum, and there is still some residual amount left, which is most apparent as the swirling motion in the bulk of the toroidal ring. In figure 7, the centre of the drop dries out, and the numerical method stops at this singularity in the velocity field. When the initial velocity is slightly lower, for example $V=35 \mathrm{~cm} \mathrm{~s}^{-1}$, dry-out does not occur, and the drop then begins to rise at the centre as the kinetic energy increases. When the drop rises further, kinetic energy starts to decrease again. 

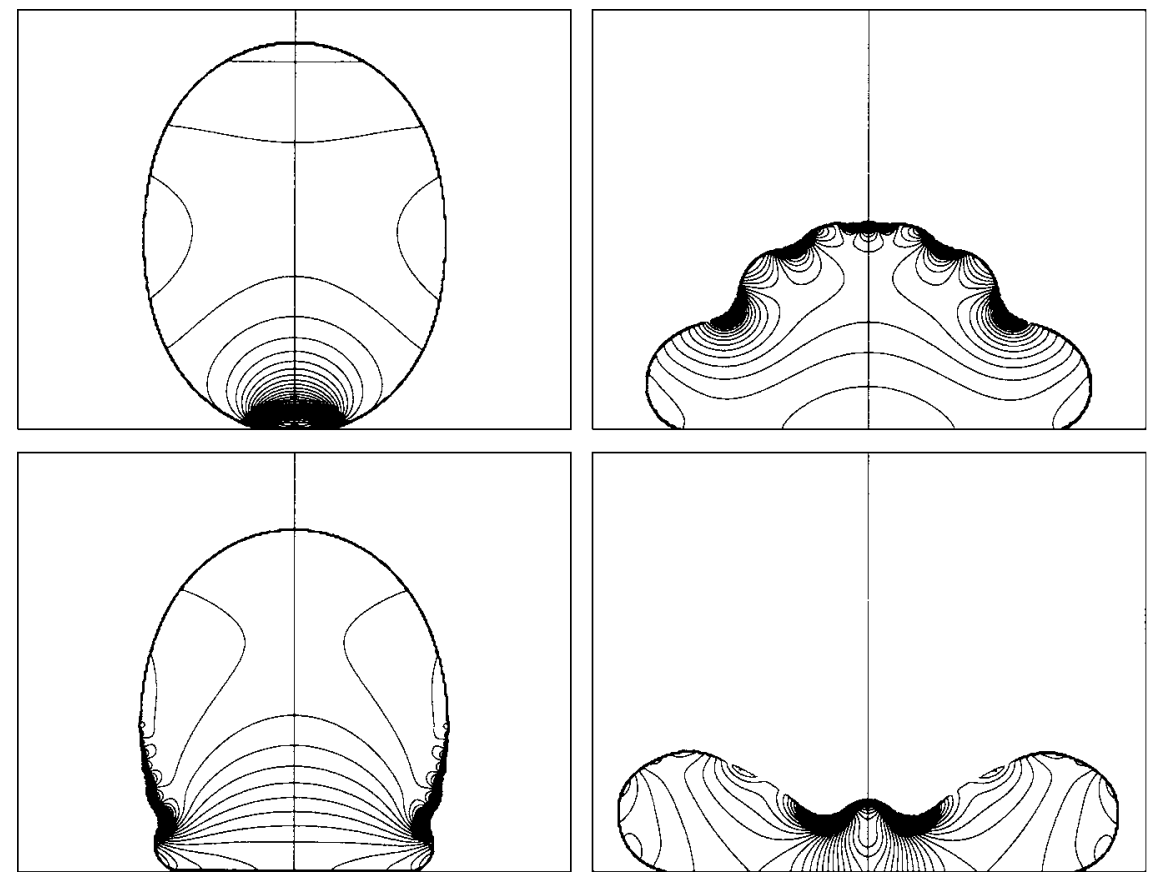

Figure 6. Pressure fields corresponding to the drop evolution of figure 4.

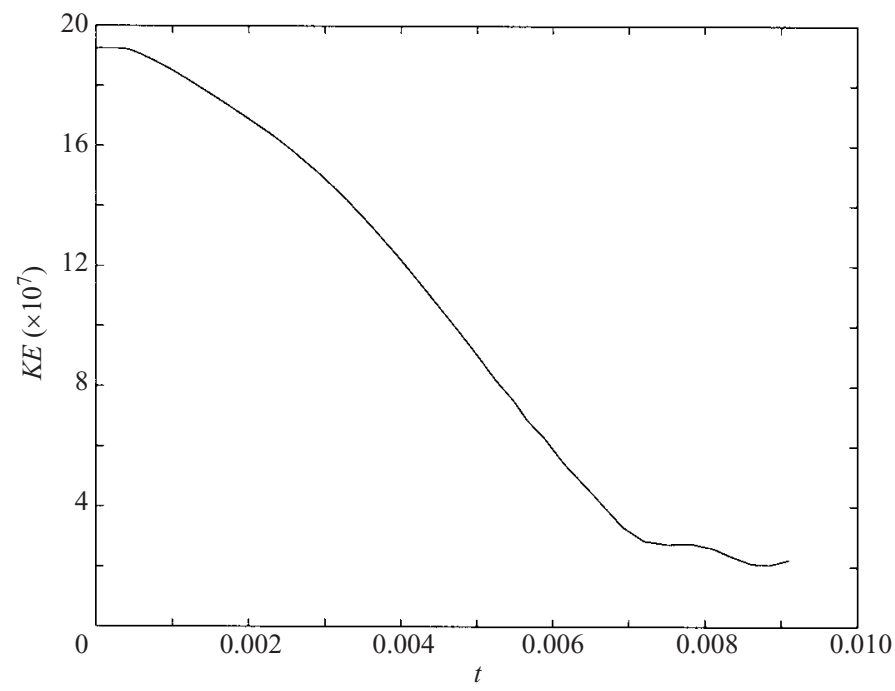

FIGURE 7. Kinetic energy vs. time in MKS units, for the simulation of figure 4. Mesh $\Delta x=0.4 \dot{6} / 256$.

These results may contribute to an understanding of why a simple criterion overpredicts the experimentally observed horizontal spread: when the maximal radius is estimated by balancing the initial kinetic energy with surface energy, the estimate leaves out the small amount of kinetic energy present in the toroidal vortical motion. 
(a)

(b)

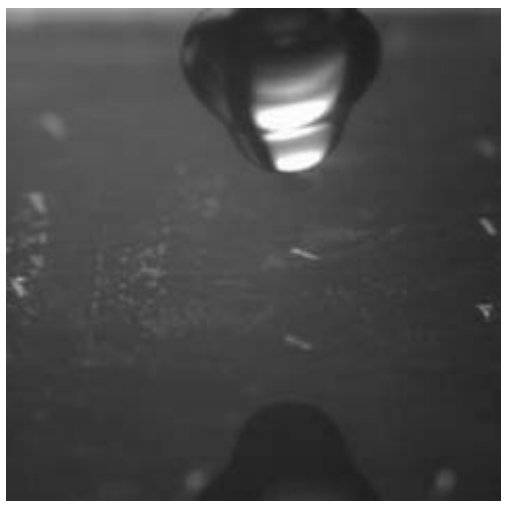

FIGURE 8. Upon total rebound, the drop typically takes a top-heavy shape. (a) Numerical simulation with velocity vectors and pressure contours for conditions of figure 4 except that $V=35 \mathrm{~cm} \mathrm{~s}^{-1}$. (b) Experimental photograph taken from above the drop at an angle for a typical total rebound. $V=1 \mathrm{~m} \mathrm{~s}^{-1}, R=1 \mathrm{~mm}$, time from impact $57 \mathrm{~ms}$.

As discussed in the introduction, a super-hydrophobic surface allows the drop to ball up upon recoil and rebound. For a normal surface, recoil leaves a residue with droplets possibly emitted by a central mass. After it lifts off, the drop evolves to a top-heavy mushroom shape as shown in figure 8(a). Experimental photographs for the total rebound for the conditions of figure 1 are not available, but are qualitatively similar to figure $8(b)\left(V=1 \mathrm{~m} \mathrm{~s}^{-1}, R=1 \mathrm{~mm}\right)$. Figure 9 shows the evolution of the kinetic energy for a situation where rebound occurs.

\section{Maximal spreading radius}

When an initially spherical drop of radius on the order of millimetres spreads out at impact, the maximal spreading radius is on the order of a centimetre. At speeds on the order of metres per second for our water drop,

$$
\text { We } \gg 1 \text {, }
$$

which means that the surface energy of the drop prior to impact is negligible in comparison to its kinetic energy. We denote the initial radius by $R_{0}$ and impact speed by $V$. For simplicity, the drop shape at maximal spreading is approximated by a disk 


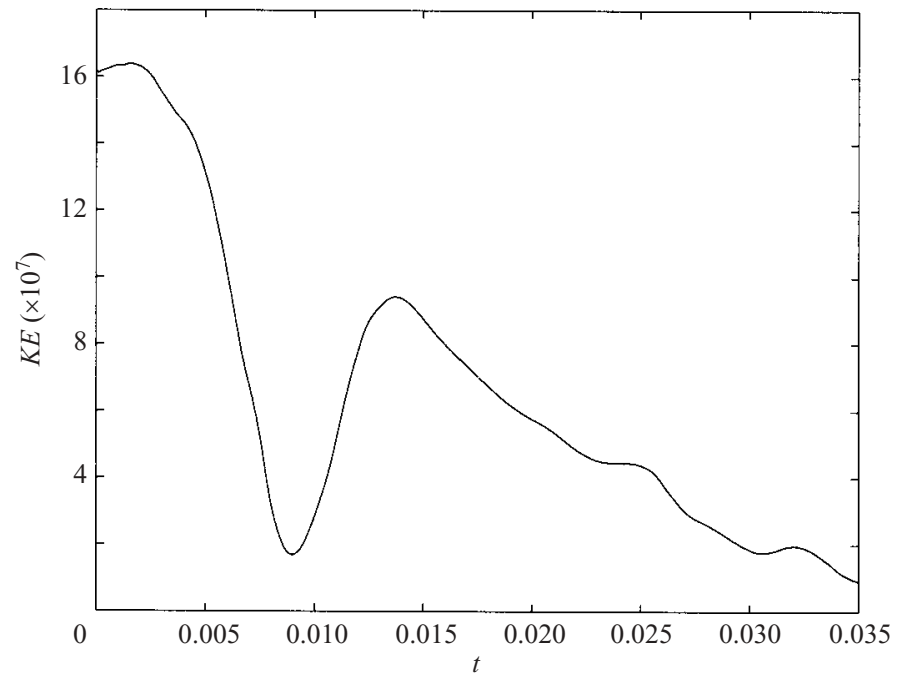

FIGURE 9. Impact of an ellipsoidal drop of radii $R_{\text {horiz }}=0.15, R_{x}=0.24 \mathrm{~cm}$ at initial velocity $V=38 \mathrm{~cm} \mathrm{~s}^{-1}$ started touching the ground. Kinetic energy vs. time, computed in $[0,0.45] \times[0,0.675] \mathrm{cm}^{2}$ with mesh $\Delta r=\Delta x=0.45 / 256$.

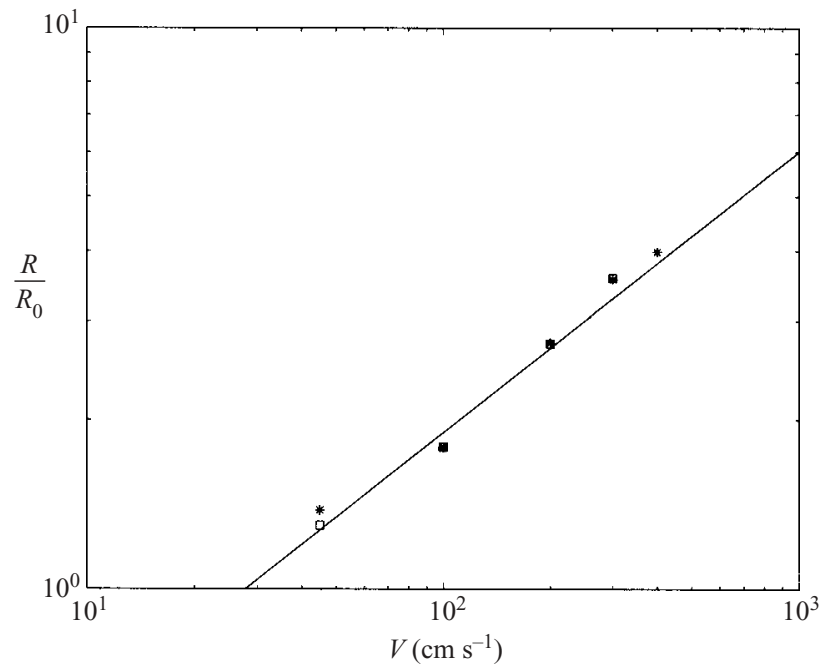

FiguRE 10. Dimensionless maximal radius $R / R_{0}$ vs. $V \mathrm{~cm} \mathrm{~s}^{-1}, R_{0}=0.8 \mathrm{~mm}$. Comparison of numerical (*) and experimental results $(\square)$. Numerical data are computed on a $256 \times 256$ mesh on a square computational domain (length of side $8 / 3 R_{0}$ for impact speeds 45 and $100 \mathrm{~cm} \mathrm{~s}^{-1}$, $4 R_{0}$ for speeds 200 and $300 \mathrm{~cm} \mathrm{~s}^{-1}$, and $6 R_{0}$ for $400 \mathrm{~cm} \mathrm{~s}^{-1}$ ). The data follow approximately a line of slope 0.5 .

of radius $R$ and thickness $e$; the use of this approximation in the case of central dry-out as in figure 2 is rough, but the volume difference between the toroidal shape and the disk is small.

The simplest way to estimate the maximal spreading radius is to assume that the kinetic energy in the drop is converted to surface energy. At the point where the disk has reached is maximal radius, the surface energy is proportional to $\gamma R^{2}$. We balance 
this with the kinetic energy at impact (which is proportional to $\rho V^{2} R_{0}^{3}$ ) to obtain

$$
R \sim R_{0} W e^{1 / 2},
$$

or $R \sim V$.

Richard (2000) obtained experimental data on the maximal radius vs. $V$ for a water drop of a different initial radius, $0.8 \mathrm{~mm}$. The dependence of the maximal radius on impact speed is shown in figure 10. The numerical data are the asterisks and the experimental values are squares. The figure shows close agreement between the two results. These data scale as approximately $R \sim \sqrt{V}$. This is at variance with the theory above which would predict $R \sim V$. We believe that the main reason for this discrepancy is that the assumption of a thin disk underlying the above theoretical argument is not justified at the values of $R / R_{0}$ which are actually obtained. We note that in the limit of small impact speed, $R-R_{0}$ would be proportional to $V^{2}$, so the slope $\mathrm{d} \ln R / \mathrm{d} \ln V$ would tend to zero. Because of dry-out and associated numerical difficulties, we cannot continue our simulations into a range where the assumption that the drop spreads into a thin disk is valid.

\section{Conclusions}

When a drop of liquid impacts a super-hydrophobic surface, it evolves into a pyramid structure when the impact speed is sufficiently high and capillary waves persist over the surface of the impacting drop. In terms of dimensionless parameters, this criterion requires $\mathrm{We}>1$ and $\mathrm{We} C a<1$.

Numerical simulations are conducted for a water drop with a free-surface fronttracking algorithm, and a satisfactory comparison with the experimental photographs is achieved.

We find that at the first minimum value of kinetic energy, the drop achieves its maximal spreading radius and is toroidal, storing the remaining kinetic energy in a swirling motion in the drop. This is followed by a rebound of the entire drop when the impact speed is sufficiently high, but the kinetic energy on the rebound is smaller than the original value. We believe that this is because the drop does not return to its original shape at rebound and therefore has increased surface energy. The effect of viscous dissipation is likely to be minor at the Reynolds numbers involved.

We have determined the critical curve in the Reynolds number vs. Weber number space for the conditions under which the centre of the drop dries out. In a given experiment where the impact speed can be varied, this study shows that there is a critical impact speed beyond which the centre dries out.

We comment that the fine structure of the capillary waves may well be affected by small changes in the initial condition. It is likely that not only the drop ellipticity, but also the velocity field inside would play a role. For instance, when one does a study of a plane capillary wave oscillation or an instability, it is very important to initiate not only the interface deformation but also the velocity field. In addition to the velocity field from the possible oscillations of the drop upon its creation, there may be a contribution from air friction during the fall. Furthermore, the droplet is in a state of superposition between various modes of oscillation, as not only mode 2 is excited. The study of these issues is a worthwhile topic for future research.

This research was sponsored by CNRS, NSF-INT, NSF-DMS, NSF-CTS, ACS-PRF, NSF-SCREMS, and utilized the Illinois NCSA SGI Origin 2000. 
Acknowledgement is made to the donors of The Petroleum Research Fund, administered by the ACS, for partial support of this research.

\section{REFERENCES}

Bico, J., Marzolin, C. \& QuÉRÉ, D. 1999 Pearl drops. Europhys. Lett. 47, 220-226.

Boulton-Stone, J. M. \& Blake, J. R. 1993 Gas bubbles bursting at a free surface. J. Fluid Mech. 254, 437-466.

Bussmann, M., Mostaghimi, J. \& Chandra, S. 1999 On a three-dimensional volume tracking model of droplet impact. Phys. Fluids 11, 1406-1417.

Duchemin, L., Popinet, S., Josserand, C. \& Zaleski, S. 2001 Jet formation in bubbles bursting at a free surface. Submitted to Phys. Fluids (also at www.lmm.jussieu.fr/ zaleski/ interface_pubs.html).

Fukai, J., Shiba, Y., Yamamoto, T., Miyatake, O., Poulikakos, D., Megaridis, C. M. \& Zhao, Z. 1995 Wetting effects on the spreading of a liquid droplet colliding with a flat surface: experiment and modeling. Phys. Fluids 7, 236-247.

Fukai, J., Zhao, Z., Poulikakos, D., Megaridis, C. M. \& Miyatake, O. 1993 Modeling of the deformation of a liquid droplet impinging upon a flat surface. Phys. Fluids A 5, 2588-2599.

Hartley, G. \& Brunskill, R. 1958 Reflection of water drops from surfaces. In Surface Phenomena in Chemistry and Biology (ed. J. F. Danielli), pp. 214-223. Pergamon.

LamB, H. 1932 Hydrodynamics. Cambridge University Press.

Martin, J. 1991 Collapsing Water Drop. http://www.pacsci.org/public/education/gallery/ high_speed_photos/student_photos.html.

Nakajima, A., Hashimoto, K. \& Watanabe, T. 2001 Recent studies on super-hydrophobic films. Monatshefte fr Chemie 132, 31-41.

Neinhuis, C. \& Barthlott, W. 1997 Characterization and distribution of water-repellent, selfcleaning plant surfaces. Ann. Bot. 79, 667-677.

Oguz, H. N. 1998 The role of surface disturbances on the entrainment of bubbles. J. Fluid Mech. 372, 189-212.

Pasandideh-Fard, M., Qiao, Y. M., Chandra, S. \& Mostaghimi, J. 1996 Capillary effects during droplet impact on a solid surface. Phys. Fluids 8, 650-659.

Plesset, M. S. \& Prosperetti, A. 1977 Bubble dynamics and cavitation. Annu. Rev. Fluid Mech. 9, $145-185$.

Popinet, S. \& ZALESKi, S. 2002 Bubble collapse near a solid boundary: a numerical study of the influence of viscosity. J. Fluid Mech. 464, 137-163.

RichaRD, D. 2000 Situations de mouillage nul. PhD thesis, Universite Paris VI.

RiCHARD, D. \& QuÉRÉ, D. 2000 Bouncing water drops. Europhys. Lett. 50, 769-775.

Thoroddsen, S. T. \& Takehara, K. 2000 The coalescence cascade of a drop. Phys. Fluids 12, $1265-1267$. 